\title{
On the Transient Response of Organic Electrochemical Transistors
}

\author{
Gregório C. Faria ${ }^{* \dagger}$, Duc T. Duong ${ }^{\dagger}$, Alberto Salleo
}

Prof. G.C. Faria

Prof. G.C. Faria, Dr. D.T. Duong, Prof. A. Salleo

Keywords: Organic Bioelectronics, Organic Electrochemical Transistors, Neuron Recording

\begin{abstract}
We present a universal model for the transient drain current response in organic electrochemical transistors (OECTs). Using equivalent circuits and charge injection physics, we are able to predict the drain current in OECT devices upon application of a gate voltage input. The model is applicable to both plain and membrane-functionalized devices, and allows us to extract useful physical quantities such as resistances and capacitances, which are related to functional properties of the system. We are also able to use the model to reconstruct the magnitude and shape in time of an applied voltage source based on the observed drain current response. This was experimentally demonstrated for drain current measurements under an applied action potential.
\end{abstract}




\section{Introduction}

Organic Electrochemical Transistors (OECTs) have recently been the focus of great attention due to their ability to support both ionic and electronic conduction and, especially, to their successful application as highly-sensitive impedance biosensors [1-5]. Indeed, OECTs using poly(3,4-ethylenedioythiophene) doped with poly(styrene sulphonate) (PEDOT:PSS) have been shown to exhibit some of the highest transconductances in published literature, due to their experimentally high charge capacitance involving the whole volume of the semiconducting polymer [6,7]. Moreover, these devices operate at low voltages, which make them not only compatible with aqueous solutions, but also with biological tissues that are affected by the application of high voltages. Additionally, organic chemistry enables the syntheses of a vast palette of new polymeric materials with tunable levels of conductivity, mechanical flexibility and optical transparency for specific electronic applications $[8,9]$. The device architecture of OECTs is generally simple, allowing for easy modifications and miniaturization by standard photolithographic processes[10,11]. For instance, the gate voltage at which the maximum transconductance is reached can be tuned by changing device geometry parameters such as channel width, length and thickness[7,12]. These features have made OECTs very promising for interfacing with biological systems[5,13,14].

In terms of sensing applications, OECTs have been successfully used to probe the activity of biological species, to measure the integrity of barrier tissues and cell layers, and as a monitoring tool for both in-vivo and in-vitro neural networks. Liao et al, for instance, were able to selectively monitor uric acid and glucose levels in saliva samples using a flexible, biofunctionalized OECT structure[2]. In a different type of application, Khodagholy et al successfully obtained electrocorticography $(\mathrm{ECoG})$ measurements on epileptic rat brains with an implantable OECT device [15]. Lastly, Jimison et al demonstrated the use of OECT devices for the monitoring of the integrity of Caco-2 barrier tissues with and without tight 
junction proteins [16]. In all of these cases, changes in drain current of the OECT devices are used to detect changes in the respective biological systems.

In a typical configuration, an OECT consists of a semiconducting (or conducting) polymer channel contacted by two source-drain electrodes on a flat substrate. The active layer is then placed into contact with an electrolyte solution (e.g. $100 \mathrm{mM} \mathrm{NaCl}$ ) in which a gate electrode (e.g. $\mathrm{Ag} / \mathrm{AgCl}$ ) is immersed. Upon application of a voltage on the gate electrode the polymer channel can be oxidized or reduced, which leads to a change in the carrier concentration and thereby the conductivity of the film, which is measured using the source and drain electrodes [17]. The standard method for operating an OECT device is to apply a source-drain voltage $\left(V_{D}\right)$ to the channel and monitoring the source-drain current with and without application of a gate voltage $\left(V_{G}\right)$. A sketch of an OECT device and its transient response are shown below in Figure 1a.

The parameters that are often extracted from such measurements include the transconductance $\left(g_{m}\right)$ and the response time $(\tau)$, both of which can be modulated by the presence of physical membranes or chemical solutes in the electrolyte solution $[16,18,19]$. However, these parameters do not yield detailed insights into the specific characteristics of the active polymer channel, the solution and/or the electrochemical process occurring during device operation. It is customary to extract the impedances and capacitances of the film using a completely different method and experimental setup [7,20]. Compared to cyclic voltammetry $(\mathrm{CV})$ and impedance measurements, for example, OECT measurements can be more sensitive to ionic currents as they take advantage of the amplifying properties of the transistor. Typical charging currents are on the order of pico- to micro-amps whereas the $\mathrm{ON}$ drain currents can be as high as tens of milliamps. CV and impedance measurements are also performed in a diode-like structure and not a three-terminal, transistor structure with which OECTs operate. These measurements, while quantitative when modeled correctly, require 
times on the order of minutes thereby limiting their utility in time-resolved experiments. On the other hand, the response of an OECT with a cut-off frequency on the order of $1 \mathrm{kHz}[7]$ could be measured with a voltage pulse lasting $10 \mathrm{~ms}$ or less allowing to characterize its evolution in time (e.g. during functionalization) with sub-second resolution. In most cases, the OECT response times are used as-is for the characterization of the device response without attempting to extract quantitative information from the data.

Indeed, typically, analyses of the drain current responses only compare channel charging times and absolute current densities. Tria et al for example monitored the response time of an OECT system that contains a tissue barrier sandwiched in between the polymer channel and the gate electrode[21]. The presence of such barriers increases the response time of the device, which provides only a qualitative means to probe the barrier membrane. The charging curve of the OECT, however, is rich in information as it reflects all of the physical processes occurring in the device, at the interface with the barrier and in the electrolyte. Using all the data points available in the charging curve, it stands to reason that a quantitative analysis of these responses can provide a rapid and simple way to extract information about its surroundings using the transistor characteristics.

Previously, Bernards et al have presented a theoretical model to describe the transient current response of a plain OECT device[17]. Recently, Gentile et al have proposed a theoretical model that describe the transport of saline ions in an electrolyte under the influence of an external alternating voltage to be applied on OECT devices[22]. However, both papers were not able to apply their models to fit experimental drain currents, to quantitatively extract useful device characteristics and ionic impedances, or to reconstruct applied voltage sources using well-characterized OECT devices. As will be demonstrated below, our work lays the foundation for extracting these key device properties. 
In this work, we present a general theoretical framework for modeling the transient drain current response in OECT devices quantitatively. This physical model is shown to accurately fit experimental data and yields several device characteristics including the impedance of the polymer channel, the electrolyte, and any intervening membrane affecting the flux of ions between the gate electrode and the semiconducting polymer layer. Additionally, such thorough knowledge of the device physics allows us to accurately reconstruct the magnitude and shape in the time domain of unknown gate voltage sources. This last feature was demonstrated experimentally by applying a simulated action potential to the gate electrode of a fabricated OECT device and recovering its original shape by numerically processing the drain current response, which was distorted by the imperfect device response. As a whole, the application of the model allows for the quantitative extraction of useful membrane impedances and the reconstruction of biological voltage sources (e.g. neuron action potentials) in an accurate and efficient manner.

\section{Results and Discussion}

\subsection{Modeling the Transient Current Response}

Our model considers the transient response of the drain current $\left(I_{D}\right)$, which can be broken down into three different contributing sources as is shown below in Equation (1).

$$
I_{D}(t)=I_{0}-f * I_{G}(t)+\Delta I_{c h}=I_{0}-f * I_{G}(t) \pm g_{m} V_{c h}(t)
$$

Firstly, the steady-state drain current $\left(I_{0}\right)$ is initially constant as a function of time and depends simply on the initial conductivity of the material and the device geometry. Upon application of a gate voltage, both the gate electrode and the device channel charge capacitively as anions and cations migrate to the proper electrodes. We call this the gate 
current $\left(I_{G}\right)$, which generally takes an exponential form. Note that the gate current that flows into the channel is split between the source and drain electrodes, and the fraction of this current that is measured at the drain electrode is defined as the factor $f$. Finally, a gate voltage can oxidize (inject holes/remove electrons) or reduce (inject electrons/remove holes) the polymer film and change its conductivity. This change in conductivity leads to a change in the steady-state drain current $\left(\Delta I_{c h}\right)$ and is described by $g_{m} V_{c h}$. Here $g_{m}$ is the transconductance of the device extracted from the experimental transfer curves and represents the steady-state change in drain current $I_{D S}$ per unit change in applied gate voltage $V_{G}\left(g_{m}\left(V_{\text {in }}\right)=\frac{\partial I_{D S}}{\partial V_{\text {in }}}\right)$. Note that the change in conductivity may be positive or negative, depending on whether the majority carriers are holes or electrons and whether the channel is being oxidized or reduced. Additionally, $V_{c h}$ represents the voltage at the device channel and depends on how fast ions can charge the polymer channel. Figure 1c summarizes how these current sources contribute to the overall transient response.

While $I_{0}$ and $g_{m}$ can be easily extracted from each OECT device, $I_{G}$ and $V_{c h}$ need to be modeled using an equivalent circuit. Previously we have demonstrated that the ionic system in OECT devices can be captured using a three-element, equivalent circuit, as shown in Figure 1b[23]. In this system, the resistor $R_{d}$ and capacitor $C_{d}$ represent the resistance and capacitance of the transistor's channel, respectively, while $R_{S}$ represents the resistance of the electrolyte solution. By calculating the frequency-space impedance of the circuit and performing an inverse Laplace transform, we are able to obtain a general expression for $I_{G}$ and $V_{c h}$, and thereby $I_{D}$, as a function of time when a constant gate voltage $\left(V_{\text {in }}(\mathrm{t})=V_{0}\right.$ when $t \geq 0 ; V_{\text {in }}(\mathrm{t})=0$ when $\left.t<0\right)$ is applied:

$$
\begin{aligned}
& I_{D}(t)=I_{0}+\frac{V_{0}\left(g_{m} R_{d}-1\right)}{R_{d}+R_{S}}-\frac{V_{0} R_{d}\left(g_{m} R_{S}+1\right)}{R_{S}\left(R_{d}+R_{S}\right)} \exp \left(-\frac{R_{d}+R_{S}}{C_{d} R_{d} R_{S}} t\right) \\
& \tau=\frac{C_{d} R_{d} R_{S}}{R_{d}+R_{S}}
\end{aligned}
$$


As expected, the drain current takes the form of a single exponential with a characteristic time constant tau $(\tau)$. In the case that $R_{d} \gg R_{S}$, which is often true for most OECT devices, $\tau$ simply reduces to $C_{d} R_{s}$. We can thus from here on apply Equation (2) to fit any drain current transient response in OECT devices.

We use the model to fit drain current responses in OECT devices with $R_{s}, R_{d}$ and $C_{d}$ as fitting parameters. In Figure 1d we show a typical drain current response of an OECT device $\left(g_{m}=3.94 \mathrm{mS}\right)$ fabricated on a cleaned glass substrate along with a fit to our transient current model. The active material is a $750 \mathrm{~nm}$ layer of PEDOT:PSS, the channel lengths and widths are $6 \times 6 \mathrm{~mm}$, respectively, and a $\mathrm{Ag} / \mathrm{AgCl}$ electrode is used as the gate electrode. The entire device is immersed in a $100 \mathrm{mM} \mathrm{NaCl}$ solution. A negative voltage of $-0.1 \mathrm{~V}$ is applied between the source and drain electrodes, yielding an initial current $I_{0}$ of close to $-2.8 \mathrm{~mA}$. In this case the application of a positive gate voltage of $+0.2 \mathrm{~V}$ leads to dedoping of the polymer channel and a decrease in the channel's conductivity (i.e. $+g_{m} V_{c h}$ ). Using an $f$ factor of 0.68 , which is justified below, the fit yields circuit parameters of $R_{s}=(1.9 \pm 0.5) k \Omega, R_{d}=$ $(146 \pm 25) k \Omega$ and $C_{d}=(2.7 \pm 0.3) m F$ with an $\mathrm{R}^{2}$ of 0.998 . These values are comparable to those previously published by Rivnay et al for similar device geometries and demonstrate that the model captures the OECT response well[7]. As a side note, we would also like to point out that contact resistance effects are not included in the model but can be accounted for as follows if necessary. Contact resistances in the gate electrode will effectively increase the apparent electrolyte resistance $R_{S}$ while those at the semiconducting polymer channel/sourcedrain interfaces will effectively decrease the observed transconductance $g_{m}$. The study of the functional dependence of the contact resistance on operation conditions is beyond the scope of this study.

As is clear from the model above, the $f$ factor is required to properly fit and extract impedances from the OECT system. Although previous works have assumed $f$ to be 0.5 , we 
show below that this is not always the case[24].The extraction of the $f$ factors can be done by first measuring both the gate and drain current responses upon application of a gate voltage. We can then fit the gate current $I_{G}$ using the same method above to calculate $R_{s}, R_{d}$ and $C_{d}$. These impedances are then used to fit the drain current response with $f$ as the only fitting parameter (see Supporting Information for more details). Figure 2 shows the extracted $f$ factors under different gate and drain voltages for a PEDOT:PSS OECT (thickness of $700 \mathrm{~nm}$, $W=4 \mathrm{~mm}, L=6 \mathrm{~mm})$. The results show several key characteristics for OECT devices. Firstly, for a positive gate voltage $\left(+V_{i n}\right), f$ is measured to be roughly 0.5 for $V_{d}=0$ regardless of the magnitude of the gate voltage. This value of $f$ is to be expected since charges cannot distinguish between the source and the drain electrode when they are shorted. When $V_{d}$ is non-zero, however, $f$ begins to deviate from 0.5 . When $V_{d}<0$, for instance, the drain electrode represents a preferred pathway for positive charges that are migrating to the channel upon application of a positive gate voltage (Figure 2b) thereby explaining why $f$ drifts towards 1 as $V_{d}$ becomes more negative. Conversely, when $V_{d}>0$, the drain electrode represents a non-preferred pathway for positive charges that are migrating to the channel and $f$ drifts towards 0 as $V_{d}$ becomes more positive (Figure 2c). Note that these two trends are expected to be reversed for negative gate voltages. For a fixed drain voltage, $f$ also drifts towards 0.5 as the gate voltage increases. Finally, the $f$ factor is most likely a function of the length, width and thickness of the active channel as well as the gate-channel distance. Investigations into the effects of these geometric factors on $f$ is ongoing and will be the subject of a future publication. For the purpose of this manuscript, we point out that due to the dependencies outlined above, $f$ must be evaluated in a device at the values of gate and drain voltages that will be used during the operation of the functionalized OECT.

\subsection{Modeling Membrane-Functionalized OECTs}


The transient current model is also very useful for applications of OECTs as ionic impedance sensors. Previous work has shown that OECTs can be used to probe and monitor the characteristics of a physical membrane that is sandwiched between the gate electrode and the polymer channel, thereby modulating the ion flux in and out of the OECT[16,21,23]. The biggest limitation common to these measurements is the fact that the membrane properties are represented only by the time response of the transistor and all the information contained in a large number of data points is reduced to a single number. By applying our model, this is no longer the case and the resistances and capacitances of the entire system can be quantified. When a device is functionalized with a partially permeable membrane, the ionic system can be represented by a five-element circuit model with an extra circuit loop containing a resistor $\left(R_{b l}\right)$ and capacitor $\left(C_{b l}\right)$ to represent the blocking membrane layer (Figures 3a-b). Solving for the drain current using the same method, we obtain the following expression for $I_{D}$ :

$$
I_{D}(t)=A+B_{1} \exp \left(-\frac{t}{\tau_{1}}\right)-B_{2} \exp \left(-\frac{t}{\tau_{2}}\right)
$$

Compared to Equation (2) the drain current response for a membrane-functionalized OECT consists of two exponential forms and corresponding time constants. Specific details regarding constants $A, B_{1}, B_{2}, \tau_{1}$ and $\tau_{2}$ as a function of impedances and capacitances can be found in the Supporting Information. We label $\tau_{1}$ such that it is always greater than $\tau_{2}$ and, because the capacitance of the polymer channel is generally larger than that of the membrane, $\tau_{1}$ represents the charging time of the polymer channel. The time constant $\tau_{2}$ thus represents the charging time of the blocking membrane layer.

In Figure 3c we present simulated drain current responses for membranes of varying impedances using device characteristics extracted from References [6] and [7]. Here we use device impedances for a high performance PEDOT:PSS device $\left(R_{s}=1.8 \mathrm{k} \Omega, R_{d}=18 \mathrm{M} \Omega, C_{d}\right.$ $=150 \mathrm{nF})$ and typical supported lipid bilayers $\left(R_{b l}=10 \mathrm{k} \Omega \mathrm{cm}^{2}, C_{b l}=1 \mu \mathrm{F} / \mathrm{cm}^{2}\right)$, and an 
optimal positive gate voltage $\left(V_{i n}\right)$ of $0.275 \mathrm{~V}$ and negative drain voltage of $-0.6 \mathrm{~V}$. Those led to a maximum transconductance value of $2.7 \mathrm{mS}$ and a steady-state drain current of $-1.3 \mathrm{~mA}$. For simplicity, the factor $f$ is set to 1 due to the high negative drain voltage. The results show that the device response slows down as $R_{b l}$ increases, which matches well with previously published results [16,21]. As a proof of concept, we also analyze OECT drain current responses previously published by Jimison et al for monitoring the integrity of Caco-2 tissue barriers (Figure 3d) [16]. First, we fit our transient model to the current response of the plain device (Figure 1c in Reference [16]) and are able to extract circuit parameters of $R_{S}=$ $(4.6 \pm 0.4) k \Omega, R_{d}=(1.4 \pm 0.4) M \Omega$ and $C_{d}=(14.1 \pm 0.6) \mu F$ with an $\mathrm{R}^{2}$ of 0.975 (using a $g_{m}=0.166 \mathrm{mS}$ and $f=0.6$ based on the applied gate and drain voltages). Using Equation (3) we obtain a device response time of $\sim 65$ milliseconds. Next, we fit the current responses of the same OECT after the cell layers have been added to the system, which yields membrane impedances of $R_{b l}=(50.7 \pm 0.8) k \Omega$ and $C_{b l}=(420 \pm 50) n F$ with an $\mathrm{R}^{2}$ of 0.988 , and response times $\tau_{1}$ of $\sim 20$ seconds and $\tau_{2}$ of $\sim 1.7$ milliseconds. As was reported in the paper, this dramatic increase in response time $\tau_{1}$ is due to the ion-blocking properties of the cell layer and our technique allows for the quantification of both the ionic resistance and capacitance of the cell layer. The much shorter $\tau_{2}$ represents the charging time of the lipid bilayer membrane.

\subsection{Reconstruction of Voltage Sources from Drain Current Responses}

Finally, our transient model can also be applied to determine the shape and magnitude of any applied voltage source based on the drain current response of an OECT device. This is possible by using a complete set of functions such as the polynomial series to construct the input voltage: 


$$
V_{\text {in }}(t)=A_{0}+A_{1} t+A_{2} t^{2}+A_{3} t^{3} \ldots
$$

By calculating $I_{G}(t)$ and $V_{c h}(t)$ using $V_{i n}(t)$ as defined in Equation (5), we obtain an expression for the experimental drain current $I_{D}(t)$ as defined in Equation (1) that is a function of the coefficients $A_{n}$. This expression can be used to extract the values of $A_{n}$, thereby giving us the original input voltage $V_{\text {in }}(t)$ that generated the output current. The number of terms needed to capture the voltage source depends on the complexity of the device response and ultimately yields the functional form of the voltage source. Note that any complete set of functions may be used and the choice of the polynomial series was dictated by convenience and simplicity. It is also required that $f, R_{s}, R_{d}$ and $C_{d}$ are known for the device in order to perform this fitting routine. This constraint is not limiting as it is provided by a reference measurement prior to starting the actual experiment.

An application of significant interest is the use of OECTs as probes to measure neurons' action potentials. In this case, the flux of sodium and potassium ions across protein channels acts as a voltage source in an electrolyte solution [25] and has the general shape depicted in Figure 4a (open circles). To demonstrate how we can experimentally back calculate this voltage form, we first apply this action potential from a pulse generator to an OECT device and measure the drain current response. Note that the action potential here is not representative of extracellular variations, which can be much smaller in magnitude, but rather that measured across the cell membrane. The OECT used was first characterized using a square voltage pulse $\left(V_{\text {in }}=+0.3 \mathrm{~V}, V_{D}=-0.1 \mathrm{~V}, f=0.65\right)$ and $g_{m}, R_{s}, R_{d}$ and $C_{d}$ were determined to be $0.12 \mathrm{mS},(1.6 \pm 0.1) \mathrm{k} \Omega,(146 \pm 25) \mathrm{k} \Omega$ and $(0.22 \pm 0.02) \mu \mathrm{F}$ with an $\mathrm{R}^{2}$ of 0.985, respectively (see Supporting Information). The measured drain current (Figure 4a, open squares) in essence represents what we may observe by placing a device close to an active neuron cell. Unsurprisingly, the drain current response is very different from the input voltage. The depolarization portion (0-0.8 milliseconds), for instance, shows an inversion 
feature that does not exist in the voltage pulse, while the hyperpolarization portion (1-3 milliseconds) is much delayed in the drain current response. These differences are attributed to the fact that the device is simply not fast enough to capture the applied potential accurately. Using our model, we can represent the observed drain currents using the polynomial voltage series as an input and with parameters $A_{n}$. Figure 4a displays the reconstruction of the drain current using up to 18 polynomial terms (blue dotted line). With this information we are able to retrieve the shape of the original input voltage (Figure 4a, red solid line). Clearly the shape of the back-calculated voltage accurately matches the actual input voltage.

Our model is particularly useful because it shows that the speed of the device need not match the signal speed if the physics of the OECT is sufficiently well understood. Here we simulate what the drain current may look like for devices of different sizes (10 to $10,000 \mu \mathrm{m}^{2}$ ) using $g_{m}, R_{s}$ and $R_{d}$, and $C_{d}$ values extracted from Reference [12]. Because the input voltage is not constant in value, we need to provide a function to describe how the $f$ factor varies as a function of gate voltage. Fitting the data shown in Figure 2a for a drain voltage $V_{D}$ of $-0.1 \mathrm{~V}$, we obtain an exponential expression for $f\left(V_{i n}\right)$ (see Supplementary Information). The simulations show that device size and thereby speed can dramatically impact the observed drain current response (Figure 4b). If the device's response is as fast or faster than the voltage source, as is the case for the $10 \mu \mathrm{m}^{2}$ device, the shape of the drain current matches near perfectly with the shape of the voltage source, within the limits of the digital resolution and the noise of the measurement. As the device size increases, the drain current response begins to significantly deviate from and lag behind the input voltage. For the 1,000 and $10,000 \mu \mathrm{m}^{2}$ devices, the simulated drain currents can still follow the signal albeit with significant distortion. In fact, most OECTs known to-date will lag behind an applied action potential and the lag time is more severe for larger devices. With the model, however, this lag can be accounted for and quantified. 
These results thus demonstrate that, with a well-characterized OECT with known impedances and capacitances, our model allows for the accurate determination of any voltage source in amplitude and phase, as long as it produces a measurable output on the OECT. This model thus has great potential for the quantitative monitoring biologically active systems such as neuron action potentials, proton flux across chloroplast membranes and ion diffusion through selective ion channels.

\section{Conclusion}

In conclusion, we have developed a theoretical model that can accurately capture the transient current response of an OECT device. We show that the model fits experimental transient curves for plain and membrane-functionalized OECT devices, and allows for the extraction of useful device characteristics. These include the important $f$ factor and the electrical impedances of the membrane, the electrolyte solution and the polymer channel. These characteristics are of interest in biosensing and monitoring applications and are now easily obtained by using our model. Finally, we also demonstrate the model's ability to quantitatively measure voltage sources in aqueous environments by taking into account device non-idealities. As an example, we were able to determine the voltage shape of a neuron action potential based on observed OECT drain current responses in the case where the response from the OECT is slower than the input signal thereby severely distorting it. Thus, we have shown as a whole that our transient model empowers OECT measurements with the capability to quantitatively characterize biological systems of interest.

\section{Experimental Procedures}


Materials: PEDOT:PSS was commercially purchased from Heraeus, under the name of Clevios PH-1000, with no further modifications. All other chemicals and solvents were purchased and used without further purifications.

Thin film device fabrication: The OECTs used throughout this work consist of a PEDOT:PSS channel defined by Au source and drain electrodes. We then proceed to make a solution containing PEDOT:PSS along with 5 vol\% of ethylene glycol, 0.1 vol\% dodecyl benzene sulfonic acid and $1 \mathrm{wt} \%$ of (3-Glycidyloxypropyl)trimethoxysilane). The formulation was sonicated for 15 min prior to spin-coating. Devices were fabricated using standard lithographic methods, with sizes ranging from $10^{8} \mu \mathrm{m}^{2}$ to $10^{4} \mu \mathrm{m}^{2}$. The conductive films were obtained by drop-casting or spin-coating PEDOT:PSS solutions onto gold-patterned substrates, giving rise to thicknesses ranging from 100 to $800 \mathrm{~nm}$. After deposition, films were baked at $120^{\circ} \mathrm{C}$ for $1 \mathrm{~h}$ and immersed overnight in deionized water to remove any excess low molecular weight compounds.

Device characterization: All electrical measurements were done using a $100 \mathrm{mM}$ $\mathrm{NaCl}$ water solution as the electrolyte gated through a $\mathrm{Ag} / \mathrm{AgCl}$ electrode (purchased from Harvard Apparatus). The OECTs response of macroscopic devices $\left(\sim 10^{8} \mu \mathrm{m}^{2}\right)$ were measured using a Keithley 2600 and a customized LabView code. Fastest devices, with sizes below $10^{5} \mu \mathrm{m}^{2}$, were measured using a dual-channel waveform generator Agilent 33600 and an oscilloscope Agilent MSO9104A Infiniium.

\section{Acknowledgements}

G.C. Faria acknowledges INCT/INEO, the Fundação de Amparo à Pesquisa do Estado de São Paulo (FAPESP) and the Brazilian National Council (CNPq/Science without Borders Project) for financial support through project numbers 2013/21034-0 and 201753/2014-6. A. S. and 
D.T. D. gratefully acknowledge financial support from the National Science Foundation

(DMR award 1507826) and from the NeuroFab project at Stanford University

\section{References}

[1] H. Tang, P. Lin, H.L.W. Chan, F. Yan, Highly sensitive dopamine biosensors based on organic electrochemical transistors, Biosens. Bioelectron. 26 (2011) 4559-4563. doi:10.1016/j.bios.2011.05.025.

[2] C. Liao, C. Mak, M. Zhang, H.L.W. Chan, F. Yan, Flexible organic electrochemical transistors for highly selective enzyme biosensors and used for saliva testing, Adv. Mater. 27 (2015) 676-681. doi:10.1002/adma.201404378.

[3] C. Liao, M. Zhang, L. Niu, Z. Zheng, F. Yan, Highly selective and sensitive glucose sensors based on organic electrochemical transistors with graphene-modified gate electrodes, J. Mater. Chem. B. 1 (2013) 3820. doi:10.1039/c3tb20451k.

[4] P. Lin, F. Yan, J. Yu, H.L.W. Chan, M. Yang, The application of organic electrochemical transistors in cell-based biosensors, Adv. Mater. 22 (2010) 3655-3660. doi:10.1002/adma.201000971.

[5] J. Rivnay, R.M. Owens, G.G. Malliaras, The rise of organic bioelectronics, Chem. Mater. 26 (2014) 679-685. doi:10.1021/cm4022003.

[6] D. Khodagholy, J. Rivnay, M. Sessolo, M. Gurfinkel, P. Leleux, L.H. Jimison, E. Stavrinidou, T. Herve, S. Sanaur, R.M. Owens, G.G. Malliaras, High transconductance organic electrochemical transistors., Nat. Commun. 4 (2013) 2133. doi:10.1038/ncomms3133.

[7] J. Rivnay, P. Leleux, M. Ferro, M. Sessolo, A. Williamson, D. a. Koutsouras, D. Khodagholy, M. Ramuz, X. Strakosas, R.M. Owens, C. Benar, J.-M. Badier, C. Bernard, G.G. Malliaras, High-performance transistors for bioelectronics through tuning of channel thickness, Sci. Adv. 1 (2015) e1400251-e1400251. doi:10.1126/sciadv.1400251.

[8] L. Akcelrud, Electroluminescent polymers, Prog. Polym. Sci. 28 (2003) 875-962. doi:10.1016/S0079-6700(02)00140-5.

[9] X. Guo, M. Baumgarten, K. Mullen, Designing ??-conjugated polymers for organic electronics, Prog. Polym. Sci. 38 (2013) 1832-1908. doi:10.1016/j.progpolymsci.2013.09.005.

[10] X. Strakosas, M. Bongo, R.M. Owens, The organic electrochemical transistor for biological applications, J. Appl. Polym. Sci. 132 (2015) 1-14. doi:10.1002/app.41735.

[11] D. Khodagholy, M. Gurfinkel, E. Stavrinidou, P. Leleux, T. Herve, S. Sanaur, G.G. Malliaras, High speed and high density organic electrochemical transistor arrays, Appl. Phys. Lett. 99 (2011) 163304. doi:10.1063/1.3652912.

[12] J. Rivnay, P. Leleux, M. Sessolo, D. Khodagholy, T. Herv, M. Fiocchi, G.G. Malliaras, Organic electrochemical transistors with maximum transconductance at zero gate bias, Adv. Mater. 25 (2013) 7010-7014. doi:10.1002/adma.201303080.

[13] R.M. Owens, G.G. Malliaras, Organic Electronics at the Interface with Biology, MRS Bull. 35 (2010) 449-456. doi:10.1557/mrs2010.583.

[14] J. Rivnay, M. Ramuz, P. Leleux, A. Hama, M. Huerta, R.M. Owens, Organic electrochemical transistors for cell-based impedance sensing, Appl. Phys. Lett. 106 (2015) 43301. doi:10.1063/1.4906872.

[15] D. Khodagholy, T. Doublet, M. Gurfinkel, P. Quilichini, E. Ismailova, P. Leleux, T. 
Herve, S. Sanaur, C. Bernard, G.G. Malliaras, Highly conformable conducting polymer electrodes for in vivo recordings, Adv. Mater. 23 (2011) 268.

doi:10.1002/adma.201102378.

[16] L.H. Jimison, S.A. Tria, D. Khodagholy, M. Gurfinkel, E. Lanzarini, A. Hama, G.G. Malliaras, R.M. Owens, Measurement of barrier tissue integrity with an organic electrochemical transistor, Adv. Mater. 24 (2012) 5919-5923. doi:10.1002/adma.201202612.

[17] D.A. Bernards, G.G. Malliaras, Steady-state and transient behavior of organic electrochemical transistors, Adv. Funct. Mater. 17 (2007) 3538-3544. doi:10.1002/adfm.200601239.

[18] G. Tarabella, G. Nanda, M. Villani, N. Coppedè, R. Mosca, G.G. Malliaras, C. Santato, S. Iannotta, F. Cicoira, Organic electrochemical transistors monitoring micelle formation, Chem. Sci. 3 (2012) 3432. doi:10.1039/c2sc21020g.

[19] D.A. Bernards, G.G. Malliaras, G.E.S. Toombes, S.M. Gruner, Gating of an organic transistor through a bilayer lipid membrane with ion channels, Appl. Phys. Lett. 89 (2006) 53505. doi:10.1063/1.2266250.

[20] S. Sweetnam, K.R. Graham, G.O. Ngongang Ndjawa, T. Heumuller, J.A. Bartelt, T.M. Burke, W. Li, W. You, A. Amassian, M.D. McGehee, Characterization of the polymer energy landscape in polymer:fullerene bulk heterojunctions with pure and mixed phases, J. Am. Chem. Soc. 136 (2014) 14078-14088. doi:10.1021/ja505463r.

[21] S.A. Tria, L.H. Jimison, A. Hama, M. Bongo, R.M. Owens, Validation of the organic electrochemical transistor for in vitro toxicology, Biochim. Biophys. Acta - Gen. Subj. 1830 (2013) 4381-4390. doi:10.1016/j.bbagen.2012.12.003.

[22] F. Gentile, D. Delmonte, M. Solzi, M. Villani, S. Iannotta, A. Zappettini, N. Coppedé, A theoretical model for the time varying current in organic electrochemical transistors in a dynamic regime, Org. Electron. Physics, Mater. Appl. 35 (2016) 59-64. doi:10.1016/j.orgel.2016.05.001.

[23] G.C. Faria, D.T. Duong, A. Salleo, C.A. Polyzoidis, S. Logothetidis, J. Rivnay, R. Owens, G.G. Malliaras, Organic electrochemical transistors as impedance biosensors, Mrs Commun. 4 (2014) 189-194. doi:10.1557/mrc.2014.35.

[24] S.E.S. Jacob T. Friedlein, Mary J. Donahue, and R.R.M. George G. Malliaras, Microsecond Response in Organic Electrochemical Transistors: Exceeding the Ionic Speed Limit, Adv. Mater. (2016). doi:10.1002/adma.201602684.

[25] D. Khodagholy, T. Doublet, P. Quilichini, M. Gurfinkel, P. Leleux, A. Ghestem, E. Ismailova, T. Hervé, S. Sanaur, C. Bernard, G.G. Malliaras, In vivo recordings of brain activity using organic transistors, Nat. Commun. 4 (2013) 1575. doi:10.1038/ncomms2573. 
Figure 1. Schematic of a typical OECT device $\left(V_{d}=-0.1 \mathrm{~V}, V_{\text {in }}=+0.2 \mathrm{~V}, W=L=6 \mathrm{~mm}, \mathrm{~d}=\right.$ $750 \mathrm{~nm})(\mathrm{a})$, the corresponding ionic circuit (b), the individual current sources that contribute to the observed drain current (c) and a collected drain current measurement fitted to the transient response model $(\mathrm{d})$.

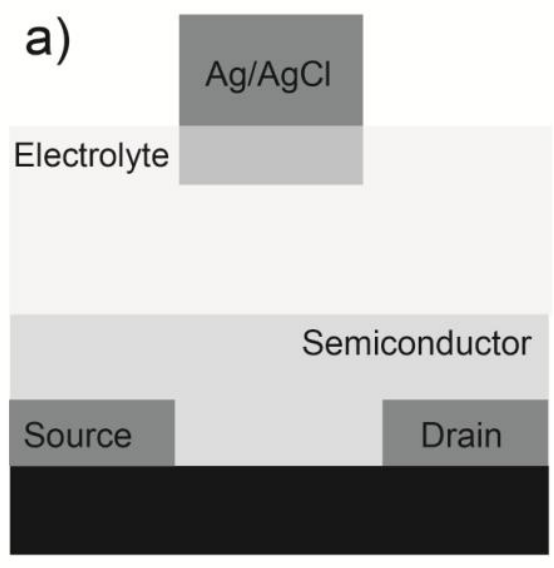

c)

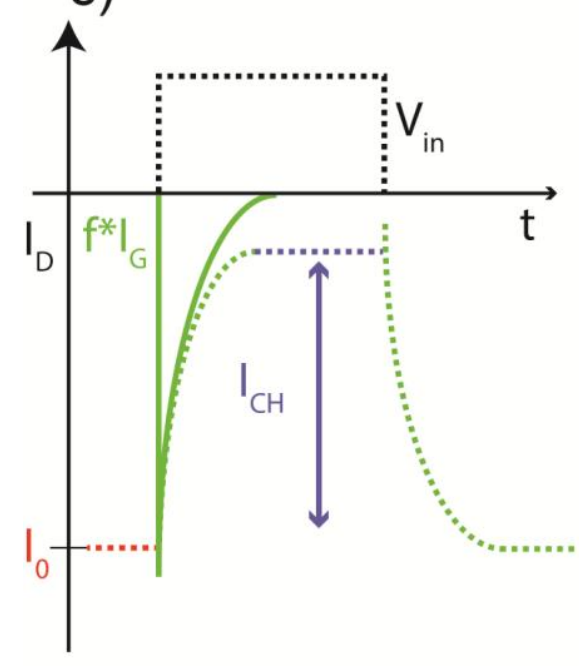

b)

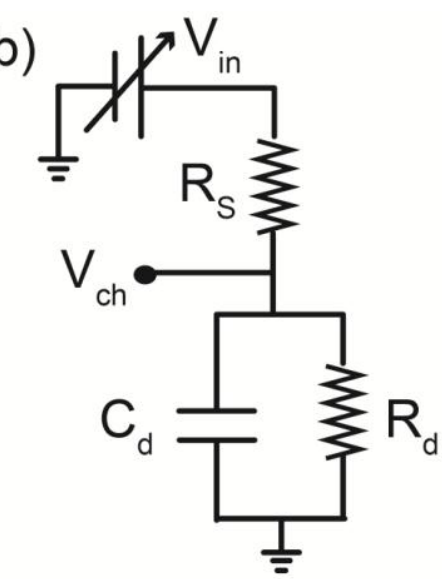

d)

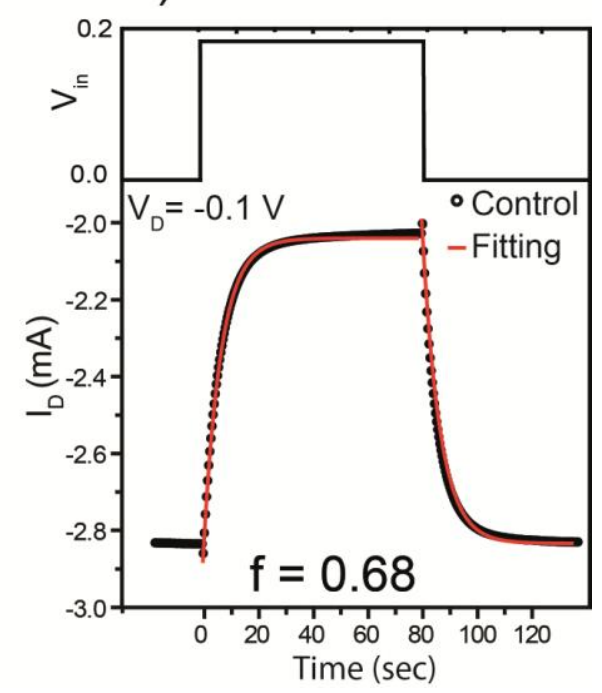


Figure 2. Summary of extrapolated $f$ factors for different drain and gate voltages and schematics showing how the gate current is distributed between the source and drain electrodes at zero (b) and negative (c) drain voltages.
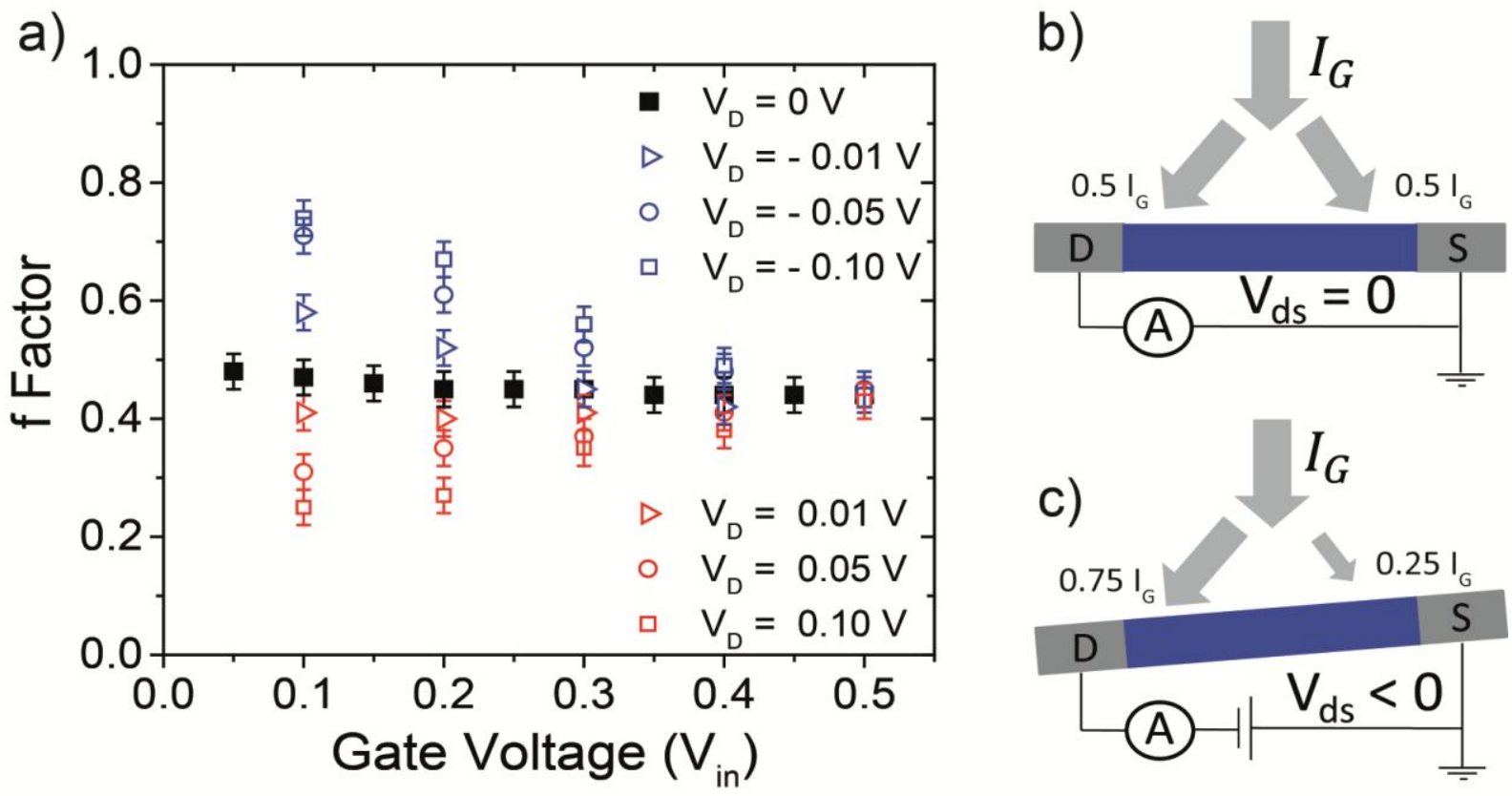
Figure 3. Schematic of a membrane-functionalized OECT device (a), the corresponding ionic circuit (b), simulated drain currents of an OECT containing membranes of varying impedances (c), and fits to experimental current transients without (solid line) and in the presence of Caco-2 cell layers (open circles), as extracted from Reference [16] (d).

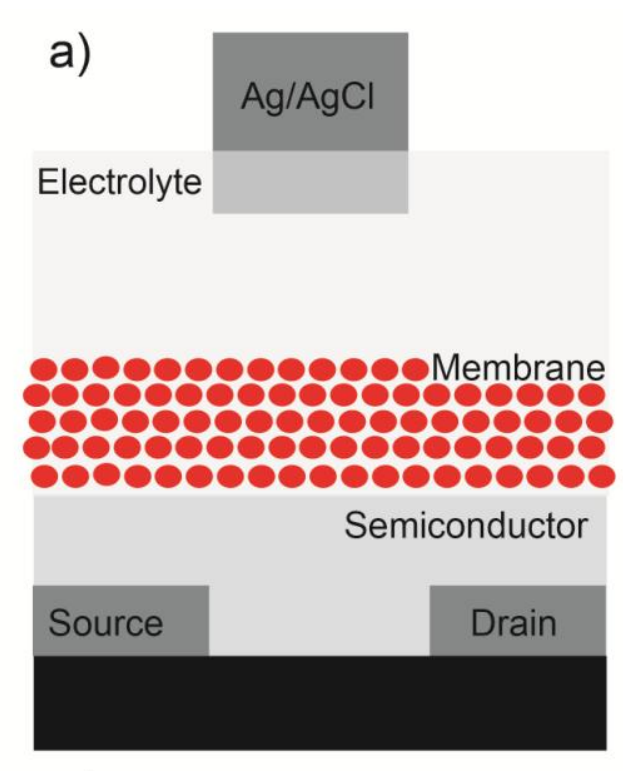

c)
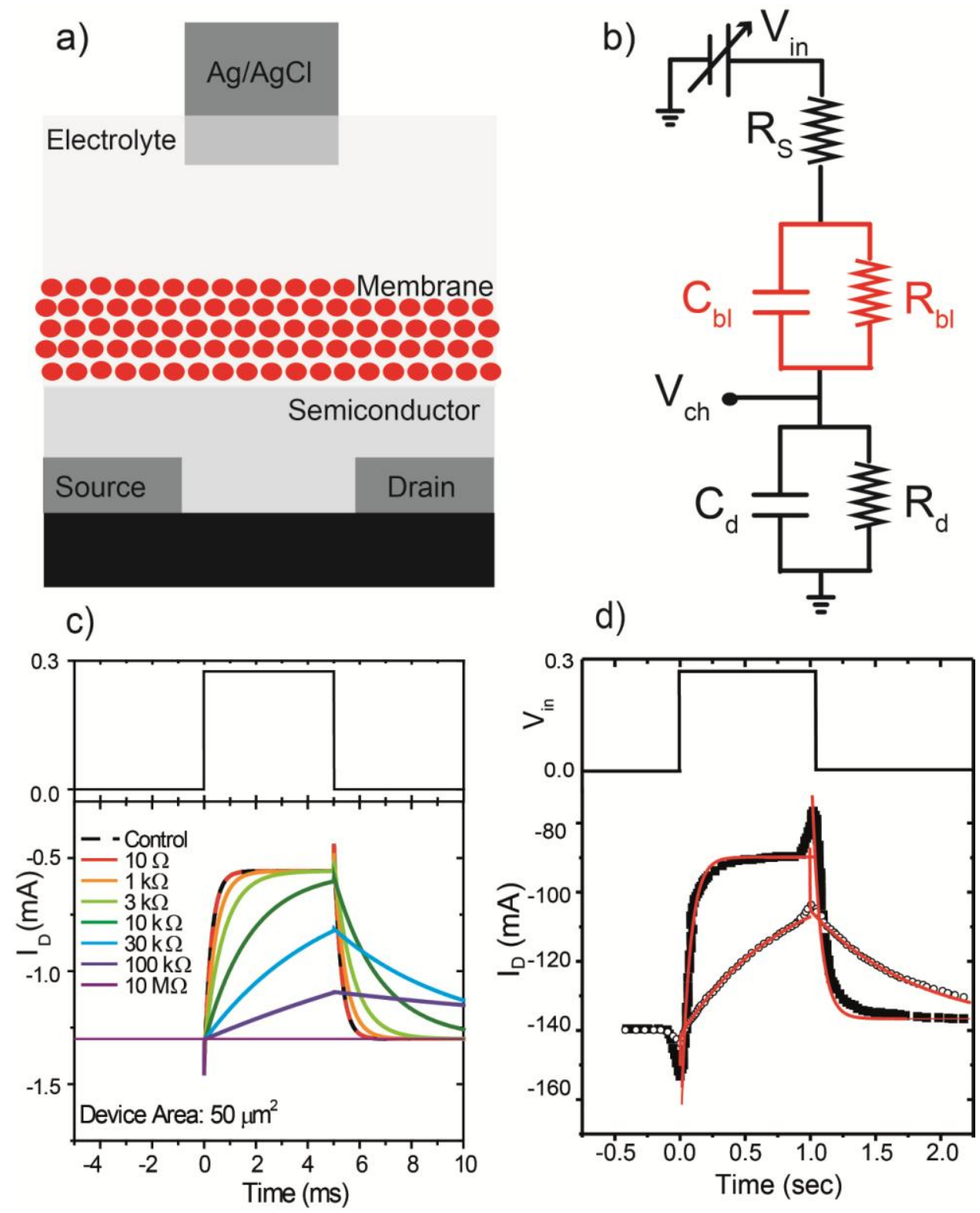
Figure 4. (a) Plots of a typical action potential (open circles) and the experimentally measured OECT response upon application of such an action potential (open squares). Using the transient model and a polynomial function of up to 18 terms, we are able to fit the drain current (blue line) and thereby reconstruct the input voltage form (red line). (b) Simulated drain currents for $10 \mu \mathrm{m}^{2}$ (red), 1,000 $\mathrm{m}^{2}$ (blue) and 10,000 $\mu \mathrm{m}^{2}$ (black) devices using parameters extracted from Reference [12].

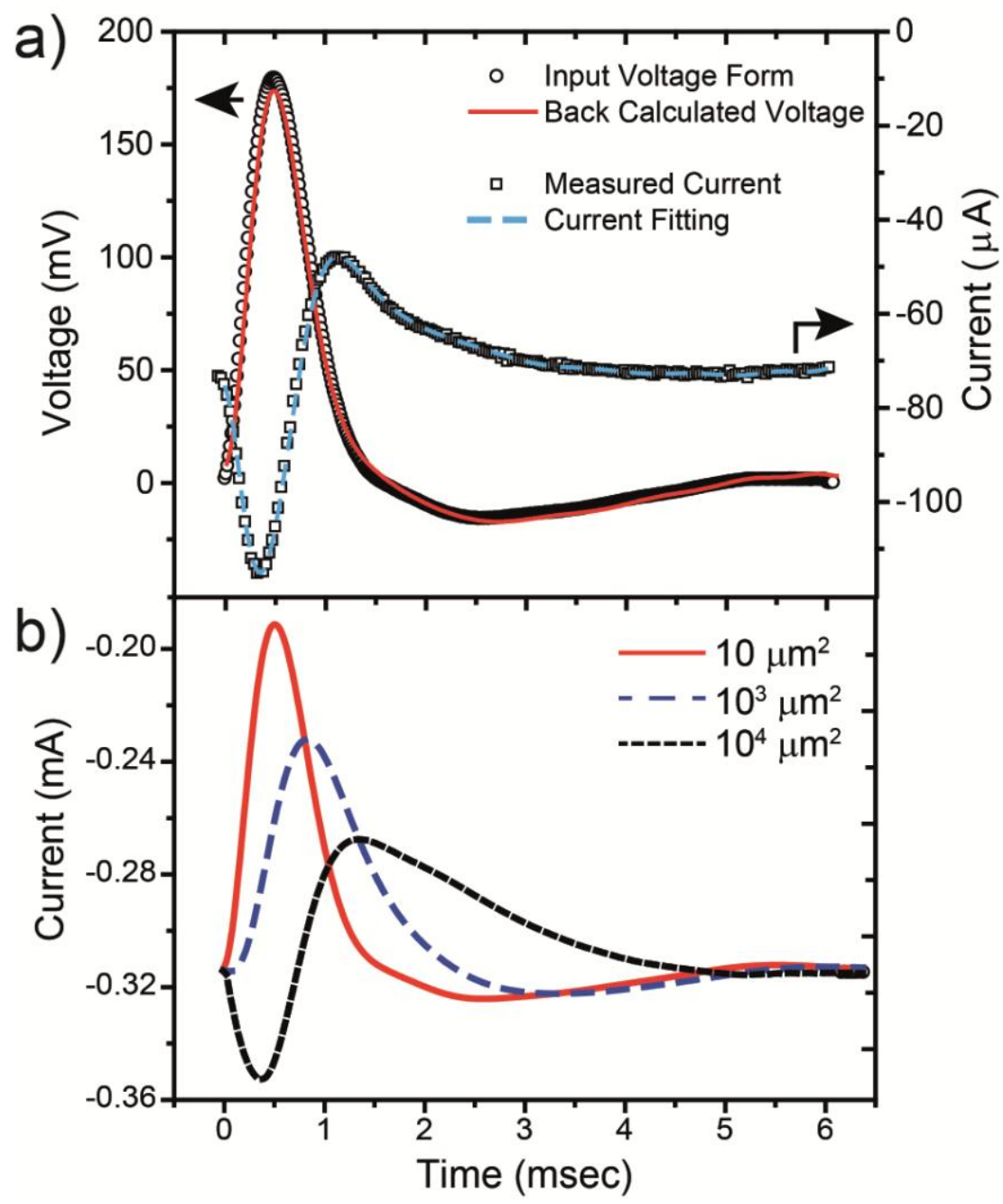



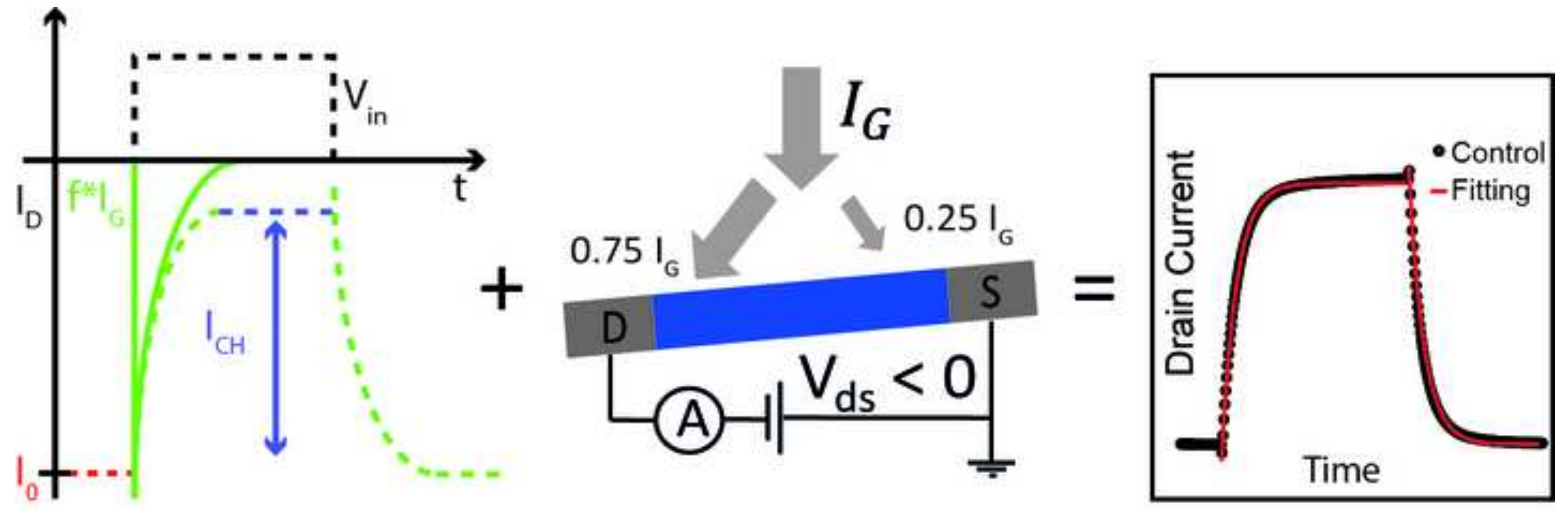